\title{
Viewpoint-dependent Priming Effects in the Perception of Human Actions and Body Postures
}

\author{
Anja Daems and Karl Verfaillie \\ University of Leuven, Belgium
}

\begin{abstract}
The identification of human actions and body postures viewed from different viewpoints was examined in four long-term priming experiments with static pictures of a human model. In Experiments 1 and 2 participants had to name or describe the pictures, and in Experiments 3 and 4 participants had to decide whether the pictures showed a possible or impossible body pose. Reliable priming effects were obtained only when priming and primed action or pose shared the same in-depth orientation (Experiments 1 and 4 ) and left-right reflection (Experiments 2 and 3 ). Having seen the same action or pose in a different orientation did not reliably facilitate identification performance later on. Also, there was no priming for poses that are impossible to perform with a human body, not even when an identical same-view prime was used. These findings suggest that the stored representations that mediate the identification of human actions and postures are viewpoint specific.
\end{abstract}

One kind of stimulus that virtually all human observers encounter numerous times in their lives is other human beings, engaged in many different actions, resulting in an even greater variety of body postures. Identifying these actions and body postures must therefore be a major task of the human visual system. This task is not straightforward, though. Depending on the orientation and position of the acting body relative to the observer, different instances of a

Requests for reprints should be addressed to A. Daems, Laboratory of Experimental Psychology, University of Leuven, Tiensestraat 102, 3000 Leuven, Belgium. Email: anja.daems@psy.kuleven.ac.be

This research was supported by a Grant from the Katholieke Universiteit Leuven, the Inter University Poles of Attraction Convention No. P4/19, the Concerted Research Effort Convention No. GOA 98/1, and by the Flemish Fund for Scientific Research, of which Karl Verfaillie is a research associate.

We thank Peter De Graef, Andreas De Troy, Paul van Diepen, Christian Lamote, and Filip Germeys for their help in constructing the stimuli and programming the experiments and Glyn Humphreys, Rebecca Lawson, and an anonymous reviewer for their comments on an earlier version of this text. 
particular action or pose may produce very different retinal images, but will have to receive identical labels nevertheless. For example, the action commonly known as "running" must be recognized as such, whether the orientation of the body is to the left, to the right, towards, or away from the observer.

The problem the visual system faces here is of course similar to the viewpoint-invariance problem in object recognition (as distinct from action recognition). Recent theories concerning the identification of objects viewed from different viewpoints, roughly divide into two classes. One proposal (e.g. Biederman, 1987; Marr \& Nishihara, 1978) states that a viewpointindependent description of the encountered object is constructed and matched against a memory representation stored in the same view point-independent format. An alternative proposal (e.g. Tarr, 1995; Tarr \& Pinker, 1989, 1990) says that separate representations for different views of an object are stored in memory. If a particular input view does not match an already existing representation, it is transformed (e.g. by a process of mental rotation) into the closest known view.

Evidence for viewpoint specificity of stored object representations was found in matching, sequential viewing, and naming tasks and in short-term and long-term priming studies. First, the response latencies to match two sequentially presented views of an object were larger when different views were presented than when the same views were shown (e.g. Ellis \& Allport, 1986; Ellis, Allport, Humphreys, \& Collis, 1989; Lawson \& Humphreys, 1996). Second, object recognition under sequential viewing conditions improved when a sequence of briefly presented views was ordered so that the object appeared to rotate in depth, relative to a random sequence of the same views (Lawson, Humphreys, \& Watson, 1994). Third, familiar objects in unfamiliar imageplane orientations (Jolicoeur, 1985, 1988, 1990) or novel objects in unfamiliar image-plane (Tarr \& Pinker, 1989) or in-depth orientations (Tarr, 1995) were named slower than familiar or novel objects in familiar orientations. Fourth, in serial-choice reaction time tasks, response times (RTs) were shorter when the object in a primed trial had the same orientation as the object in the immediately preceding, priming trial (e.g. Marshall \& Walker, 1987). Finally, Srinivas found more long-term priming in an object/non-object decision task when the objects were primed with the same view than when they were primed with a view that differed more than $67^{\circ}$ (Srinivas, 1995). In a brief presentation naming task, priming across different views was more effective when an unfamiliar view primed a familiar view than the other way around (Srinivas, 1993). These latter data suggest that unfamiliar views are recognized by transforming them into more canonical ones.

In addition to viewpoint-dependent effects, some of the aforementioned studies also documented effects that point in the direction of viewpoint invariance. First, Ellis et al. $(1986,1989)$ observed an advantage for different-view matches over matches between same-name, different-exemplar 
objects. Moreover, the advantage for same-view compared to different-view matches disappeared after longer inter stimulus intervals (ISIs) or with intervening pattern masks (but also see Lawson \& Humphreys, 1996, who did find view-effects after long ISIs and intervening masks). Second, in some long-term priming studies, viewpoint-invariant effects were found in the absence of viewpoint-dependent effects. Biederman and Cooper (1991) reported that the magnitude of same-view priming was not reduced when mirror images were used as primes and concluded that object representations are independent of their left-right orientation. In addition, Biederman and Gerhardstein (1993) showed that priming was independent of other depth-orientation changes between priming and primed object as well, provided that these changes did not produce differences in the visible parts of the objects. Hence, the question whether object identification is achieved on the basis of an orientation-dependent or orientation-independent representation seems to have no definite answer yet and is still the topic of intense debate (e.g. Tarr \& Bülthoff, 1995 vs. Biederman \& Gerhardstein, 1995; also see Logothetis \& Sheinberg, 1996 for an overview).

There are a number of reasons to caution against the direct generalization of findings from experiments on the identification of non-living things to the identification of living things in the first place and human actions in the second place. First, both neuropsychological and psychological evidence suggest that visual processing of human bodies is performed by a separate body-specific representational system. For instance, in studies with visual agnosic patients, Farah and colleagues (Farah, McMullen, \& Meyer, 1991; Farah, Meyer, \& McMullen, 1996; see also Kurbat, 1997) observed that the recognition of living things is disproportionately impaired compared to the recognition of non-living things. Another notable phenomenon is the autotopagnosia syndrome. Patients suffering from this syndrome are unable to locate body parts on their own and other human bodies (Sirigu, Grafman, Bressler, \& Sunderland, 1991), but seem to have no trouble locating parts of complex objects other than bodies (Ogden, 1985). In a series of experiments with normal human participants, Reed and Farah (1995) found that moving a part of one's own body facilitates visual detection of changes in the same part of another person's body, but not in spatially comparable parts of complex three-dimensional forms. Several other studies with normal subjects also documented differences between the perception of body parts and the perception of other objects. These studies include, among others, Parsons' (1987a, 1987b, 1994; Parsons et al., 1995; see also Cooper \& Shepard, 1975) work on handedness judgements of visually presented body parts, Shiffrar and Freyd's (1990, 1993) experiments on the perception of apparent motion of the human body, and Shiffrar, Lichtey, and Heptulla Chatterjee's (1997) study of the perception of biological motion across apertures. All these results suggest that the notion of a body schema, conceived of as a representation exclusively committed to 
the spatial relations between body parts, appears to be appropriate for characterizing both normal as well as brain-damaged participants' understanding of human bodies.

Second, given the fact that object and action recognition serve different computational goals (in the sense of Marr's computational theory, 1982), the issue of viewpoint invariance acquires an extra dimension in the case of action identification. Due to their non-rigid nature, human bodies can have an infinity of possible 3D manifestations, that can all project onto many different $2 \mathrm{D}$ retinal images. To categorize a stimulus object as a human body (which is the purpose of basic-level object recognition), the visual system must transcend the specific 3D arrangement of the body parts (and the 2D projection of this arrangement, either by accessing a viewpoint-invariant or one of several, linked viewpoint-dependent representations). Identifying a body action or pose, on the other hand, also involves transcending the particular 2D projection of the body pose, but does not necessitate abstracting away from the spatial relations between the parts. On the contrary, action or pose identification boils down to categorizing a particular 3D manifestation of a human body as a specific pose or a specific phase of a certain action. The question then becomes whether the representations that are computed to match the stored models of body actions or poses are orientation dependent or not.

Neurophysiological as well as psychological evidence suggests that the representations that mediate the recognition of the human body as a basic-level object are viewpoint specific. First, Perrett et al. (1989; Wachsmuth, Oram, \& Perrett, 1994) conducted single-cell recordings and localized cells in the temporal cortex of monkeys that are exclusively responsive to faces or moving bodies in particular orientations. Cells displaying view-specific coding were found to be common, whereas cells displaying view-general coding were rare. Second, there is abundant evidence (e.g. Bertenthal \& Pinto, 1993; Shiffrar, Lichtey, \& Heptulla Chatterjee, 1997; Sumi, 1984) that inverting a moving human body shown under point-light conditions disrupts the interpretation of the stimulus as a human figure. Third, Verfaillie and colleagues also used moving point-light walkers in a series of short-term priming (Verfaillie, 1993) and transsaccadic-integra tion experiments (Verfaillie, 1997; Verfaillie, De Troy, \& Van Rensbergen, 1994) to examine the effects of depth rotations. Priming effects were found only when the primed walker and the immediately preceding priming walker had the same in-depth orientation, regardless of, for instance, their position in the visual field or the direction of articulatory motion. Likewise, saccade-contingent changes in the in-depth orientation of an upright walker were highly detectable, whereas position changes were hardly noticed. Together, these findings suggest that the recognition of a walking figure is accomplished by accessing an orientation-dependent representation. This representation is abstract with respect to certain other characteristics of the figure (e.g. position) and is therefore high level. 
The focus of the present article is on the representations underlying action and pose identification rather than object identification. As mentioned previously, action and pose identification comes down to categorizing different configurations of the same body parts as different body postures or action phases. In the experiments reported later, the representations mediating this categorization process are examined using a long-term priming paradigm (see also Olofsson, Nyberg, \& Nilsson, 1997). Priming refers to the observation that prior viewing of a stimulus facilitates recognition later on. The effect can last over a relatively long period of time and presumably results from the persistent activation of the pre-semantic representations that mediate recognition (e.g. Riddoch \& Humphreys, 1987; Schacter, Cooper, \& Delaney, 1990). As such, characteristics of these representations are assumed to modify priming effects, which means that, if the stored representations of human actions and body poses are orientation dependent, priming effects should be orientation dependent as well. This hypothesis was examined in four experiments in which participants had to recognize static pictures of various actions and body poses. In Experiments 1 and 2 the stimuli had to be named or described and in Experiments 3 and 4 participants had to decide whether a specific configuration of body parts constituted a possible pose or not.

\section{EXPERIMENT 1}

In the first experiment, two different-view pictures of the same action were used to explore the effects of changes in depth on long-term priming. Most of the pictures showed one human being performing an everyday action with or without the help of an instrument. The remaining pictures displayed two humans acting together. The main actor in the pictures was either facing straight ahead or to the right. Participants had to respond by writing down short descriptions of the pictured actions, first in a priming and then in a primed block. The actions in the primed block were briefly presented, either in a different view or in the same view as in the priming block. If the stored representations mediating the identification of human body postures are orientation specific, same-view primes should be more effective in increasing identification performance than different-view primes.

\section{Method}

Participants. Thirty-two undergraduate psychology students participated in the experiment. They received course credit for their participation. All participants had normal or corrected-to-normal vision. 
Stimuli and Apparatus. The stimuli were two sets of 32 fully coloured pictures of different actions performed by one or two human models. In the first set the same model was always facing straight ahead. In the second set the model who was facing straight ahead in the first set was now oriented approximately $75^{\circ}$ to the right (from an observer or the camera's point of view). The pictures were produced by means of a 3 CCD colour camera and an IBM-compatible computer equipped with an ATVista $4 \mathrm{M}$ videographics adapter. There were 8 pictures showing two humans acting together (dancing, squatting down back to back, shaking hands, walking on hands while someone holds feet, carrying someone in arms, carrying someone on back, sitting on someone's lap, boxing), 12 pictures showing one human doing something with an instrument (taking photographs, shovelling, watering flowers, painting, reading book, opening umbrella, brushing hair, playing with hula hoop, drinking from a bottle, knitting, playing tennis, hammering), and 12 pictures showing one human doing something on his own without using any instruments (walking on knees, bending down, walking, doing push-ups, crawling on hands and knees, crawling on hands and feet, hopping, squatting down, sitting cross-legged, doing sit-ups, lying on back with feet high in the air, lying on back with feet walking in the air just above the ground). Examples are shown in Figure 1. In addition, five filler stimuli and six practice stimuli were used. The filler stimuli were made as described earlier and the practice stimuli were digitized pictures of actions taken from magazines. All pictures were generated by a computer equipped with an ATVista graphics system and displayed on a colour screen. In an upright pose the human body subtended approximately $12 \mathrm{~cm}$ on the screen $\left(9.7^{\circ}\right.$ with a $70 \mathrm{~cm}$ viewing distance).

Procedure. Both stimulus sets were divided into four groups of eight stimuli. These groups were rotated across participants in such a way that each stimulus appeared equally often in every possible combination of orientations in priming and primed block. Each participant saw 16 stimuli in the same-view and 16 stimuli in the different-view condition. In the same-view condition half of the stimuli were side views and the other half were straight-ahead views in both the priming and the primed block. In the different-view condition, half of the stimuli were side views in the priming block and straight-ahead views in the primed block, and the other half were straight-ahead views in the priming block and side views in the primed block. In the first phase of the experiment (priming block) all participants started with the practice stimuli, followed by a random presentation of the 32 priming stimuli. They began each trial by pushing a key on a keyboard. A fixation cross then appeared in the centre of the screen for $1 \mathrm{sec}$ followed by a $2 \mathrm{sec}$ presentation of a picture. Participants had to write down the name or a description of the pictured actions. After a five-minute break, participants started the second phase (primed block) with the practice stimuli, followed by a random presentation of the 32 primed and 5 filler stimuli. 

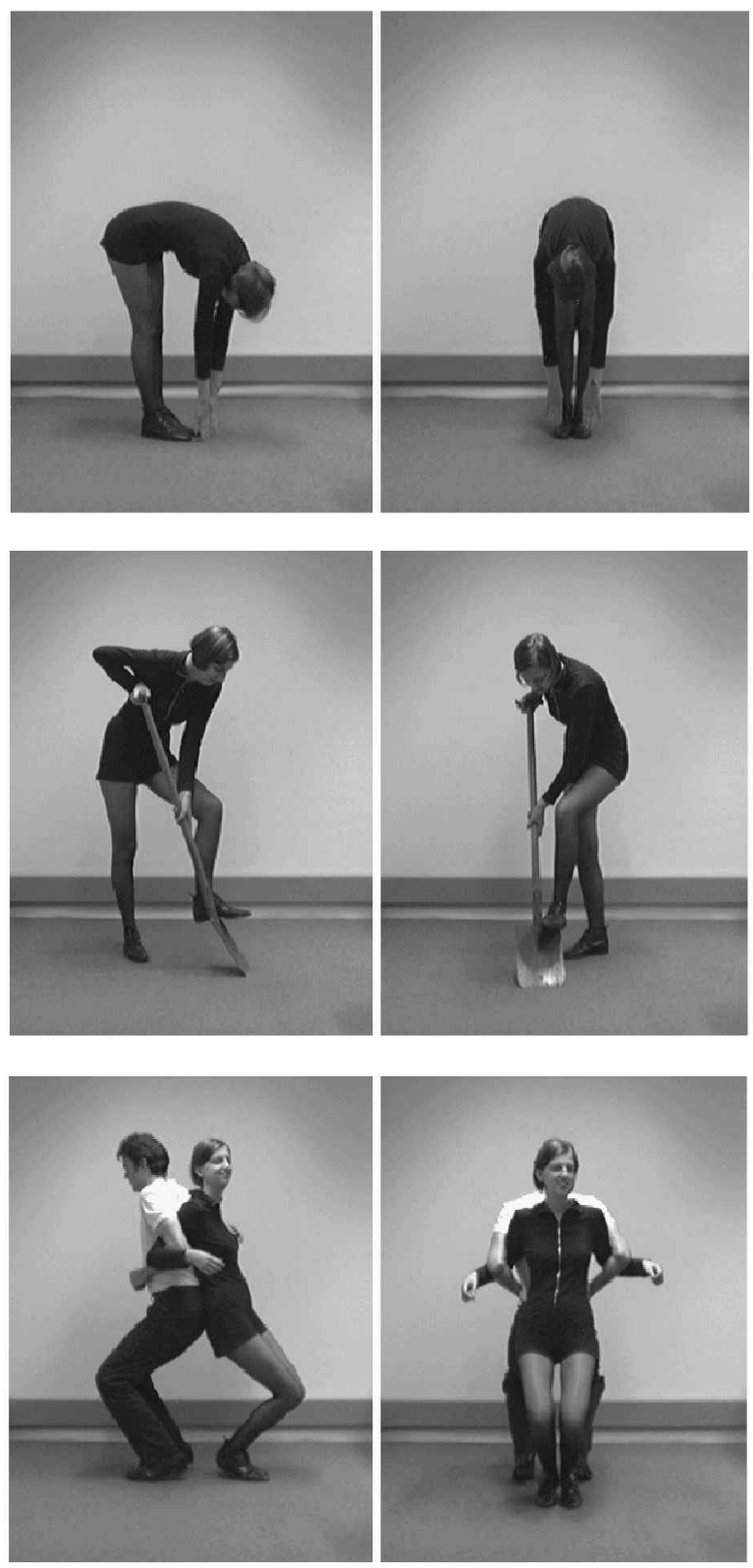

FIG. 1. The side view and the straight-ahead view of the actions "bending down", "shovelling", and "squatting down back to back". 
The procedure was the same as in the first phase, except for the presentation time of the stimuli. Each stimulus was presented for $60 \mathrm{msec}$ followed by a $500 \mathrm{msec}$ presentation of a mask. The mask was created by cutting up small sections of the pictures and pasting them together haphazardly. One experimenter scored the names or descriptions in the short-presentation primed block by comparing them to the names and descriptions in the long-presentation priming block, which were all acceptable descriptions of the portrayed actions. The primed block descriptions of the actions were considered correct when there was an exact match with the priming block descriptions. While scoring, the experimenter was unaware of the prime condition the stimuli were in.

\section{Results and Discussion}

The percentage of correctly recognized primed actions per condition are displayed in Table 1.

A repeated measures ANOVA with primed view (facing to the side or facing straight ahead) and prime condition (same or different view) as within-subjects variables and subject group (which group of participants received which stimuli in which condition) as between-subjects variable revealed two significant main effects. The percentage of correctly identified actions in the primed block was larger when the same view was presented in the priming block than when a different view was shown, $F_{1}(1,28)=32.54, M S E=0.0205, p<.0001$, and the side views were recognized more often than the straight-ahead views, $F_{1}(1,28)=65.98, M S E=0.0157, p<.0001$. Prime condition and primed view did not interact significantly, $F_{1}(1,28)=2.93, M S E=0.0202, p>.09$. The difference in identification performance between the same-view and the different-view prime condition was tested separately for each primed view with Dunn's multiple comparison procedure (Kirk, 1995, pp. 137-140, 282). Both contrasts were significant: $t D_{1}=5.91, M S E=1.61, p<.01$, for the side-facing primed views and $t D_{1}=2.59, M S E=2.46, p<.05$, for the straight-ahead primed views.

TABLE 1

Mean Recognition Percentages of Primed Actions and Poses as a Function of Prime Condition and Primed View (Experiment 1)

\begin{tabular}{lcc}
\hline & \multicolumn{2}{c}{ Prime Condition } \\
\cline { 2 - 3 } Primed View & Same View & Different View \\
\hline Right facing & 69 & 50 \\
Straight ahead & 47 & 37 \\
\hline
\end{tabular}


The results of the subject analysis were confirmed by a stimulus analysis with prime condition and primed view as within-stimuli variables and stimulus group (which stimulus list was shown to which participants in which condition) as between-stimuli variable. There was a significant main effect of prime condition, $F_{2}(1,28)=28.91, M S E=0.0231, p<.0001$, and of primed view, $F_{2}(1,28)=6.98, M S E=0.1481, p<.02$, and no significant interaction between both variables, $F_{2}(1,28)=3.29, M S E=0.0180, p>.08$. The difference between the same-view and the different-view prime was significant for both the side-facing, $t D_{2}=4.50, M S E=2.78, p<.01$, and the straight-ahead primed view condition, $t D_{2}=3.52, M S E=1.33, p<.01$. In the remainder of this paper all subject and stimulus analyses will be reported together, using subscript 1 for the subject analyses and subscript 2 for the stimulus analyses.

These results show that action identification performance is better with same-view primes than with different-view primes. The main effect of primed view and of prime condition and the absence of an interaction effect demonstrate that, although the straight-ahead views are more difficult to recognize than the side views, the effect of orientation changes is not altered.

Along with Biederman and Gerhardstein (1993), one could argue that different-view priming was hampered by the fact that the two different views of the actions and postures in Experiment 1 did not always share the same visible body parts. However, given that orientation changes almost inevitably lead to part-visibility differences, this seems a rather strange argument for a viewpoint-invariance claim. Indeed, in his more recent publications Biederman seems to have moved in the direction of viewpoint-dependent accounts of object recognition: If identification is based on viewpoint-independent representations only when there are no changes in the visible parts of an object, several viewpoint-dependent representations are needed in order to recognize all possible views. Moreover, Hayward and Tarr (1997) showed that recognition performance in sequential matching and naming tasks using novel objects was not systematically related to major changes in the visibility of parts across different views. First, systematic viewpoint dependence was observed even when changes in viewpoint did not produce changes in the visible parts, and second, the degree of viewpoint specificity depended on qualitative variations in image structure rather than on the parts themselves. Nevertheless, Experiment 2 was designed to rule out the part-visibility explanation: Part differences were minimized using mirror-image primes in the different-view condition.

\section{EXPERIMENT 2}

Half of the stimuli were the right-facing pictures of the first experiment. The other half were mirror images. This means that same-view and different-view primes only differed in left-right orientation, but not in the number, form, and 
arrangement of the body parts. If viewpoint invariance is to occur at all, it should be under these circumstances.

\section{Method}

Participants. Forty undergraduate psychology students received course credit for participation. All had normal or corrected-to-normal vision.

Stimuli and Apparatus. The stimuli were two sets of 32 fully coloured pictures of actions. In the pictures of the first set, the human model or one of the models was always facing to the right. These were the side views from Experiment 1, except for one action (drinking from a bottle), which was replaced by one of the filler stimuli (polishing shoes). The pictures of the second set were mirror images of the pictures of the first set. They were created using the ATVista Tips software. Examples are shown in Figure 2.

Procedure. Both stimulus sets were divided into four groups of eight stimuli, which were rotated across conditions and participants. Each participant saw 16 stimuli in the same-view and 16 stimuli in the different-view condition. In the same-view condition half of the stimuli were right-facing side views and the other half were left-facing (mirror-image) side views in both the priming and the primed block. In the different-view condition, half of the stimuli were side views in the priming block and their mirror-images in the primed block, and the other half were mirror-images in the priming block and right-facing side views in the primed block. The rest of the procedure was exactly the same as in the first experiment.
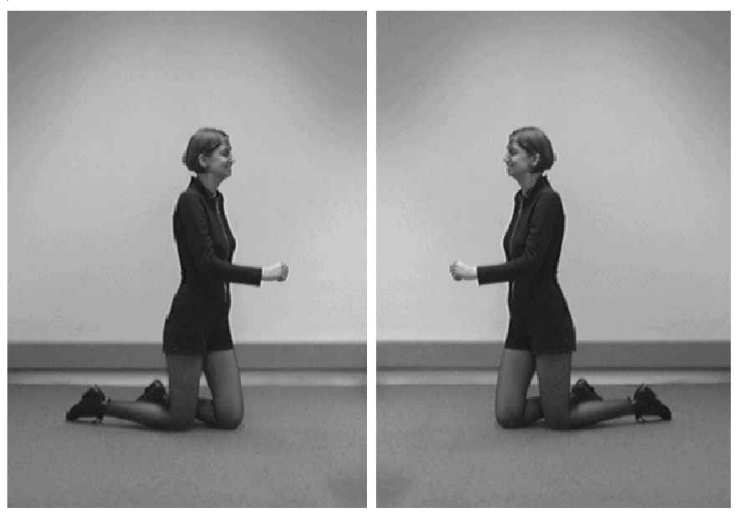

FIG. 2. The side view of the action "walking on knees" and its mirror-image. 


\section{Results and Discussion}

When actions were presented in the same orientation in priming and primed block, the average percentage of correctly identified actions in the primed block was $70 \%$. For actions presented in different orientations this percentage was only $58 \%$. A subject and a stimulus repeated measures ANOVA with prime condition (same or different orientation in priming and primed block) as within variable and subject group (or stimulus group) as between variable revealed a significant main effect of prime condition, $F_{1}(1,36)=24.90$, $M S E=0.0116, p<.0001 ; F_{2}(1,30)=24.60, M S E=0.0094, p<.0001$.

Both analyses show that mirror-image primes are less effective in increasing action identification performance than same-view primes, suggesting that the orientation-specific priming effects that were observed in Experiment 1 were not due to visibility differences in the body parts. The hypothesis that the stored representations of actions and body poses are orientation specific can thus be maintained.

Although Experiment 2 confirms the viewpoint-specificity hypothesis that was also supported by the outcome of Experiment 1, this result might seem surprising. Several long-term priming studies using objects instead of actions suggest that there is little or no cost to recognition performance with mirror-image primes as compared to identical primes (e.g. Biederman \& Cooper, 1991; Cooper, Schacter, Ballesteros, \& Moore, 1992). There is no immediate explanation for this discrepancy between object identification on the one hand and action or pose identification on the other hand. One could argue that the mirror-image prime condition in the present experiment was not a different-view condition at all. Most of the actions and poses were asymmetrical and the mirror-image of an asymmetrical pose or action phase could in fact be perceived as a different pose or action phase. Of course, this point also applies to certain asymmetrical objects, but it may be more pertinent when identification primarily concerns the configuration of the parts instead of their distinct form, as is the case with actions and body postures. If this reasoning holds, the effect that was observed here was a pose-specific or phase-specific rather than a view-specific priming effect. We will come back to this issue in the Results and Discussion section of Experiment 3.

In general, the results of Experiments and 2 support the notion of a viewpoint-dependent representation, but due to the absence of a no-prime baseline condition, there is no direct way to evaluate the possible role of a viewpoint-independent representation. However, an additional stimulus analysis on the combined results of both experiments with different-view prime type (left-facing mirror-image prime or straight-ahead view prime) as withinstimuli variable revealed an effect of prime type. The identification percentages in the mirror-image prime condition (Experiment 2) and in the straight-ahead view prime condition (Experiment 1) were $61 \%$ and 52\%, 
respectively, $F_{2}(1,30)=5.50, M S E=0.0264, p<.03 .^{1}$ The identification of a right-facing side view of an action or body pose thus benefits more from a mirror-image prime than from a straight-ahead view prime. This suggests that mirror images can produce priming as well, suggesting that the action identification process also draws upon representations that generalize over left-right reflection. Experiment 3 was designed to address this point in a more direct way.

\section{EXPERIMENT 3}

In this experiment, several methodological changes were introduced in order to further examine the effect of mirror reversal on long-term priming of body postures. First, in Experiments 1 and 2 some of the stimuli were instrumental actions, which means that a human body was not always the only object portrayed. For these actions, the observed priming effects might have been caused by the repetition of the instrument rather than of the action itself. It is even conceivable that participants remembered on which side of the picture the instrument (or the other actor in the case of the interactions) was located, thus recognizing a same-view picture more easily than a different-view picture. To avoid this problem, in Experiment 3, all actions and poses were performed by one human actor without using any instruments.

Second, a no-prime condition was included in addition to the same-view and mirror-image prime conditions, making it possible to evaluate whether mirror-image primes produce any priming at all. The observation that performance with mirror-image primes is not facilitated, would suggest that viewpoint invariance is not achieved yet on the pre-semantic level of representation that mediates the identification of human actions and poses.

Third, a forced-choice reaction-time task was used instead of a naming task. Half of the pictures in the experiment showed normal human poses, whereas the other half showed poses that were impossible to accomplish with a normal human body (the upper body of the actor was rotated $180^{\circ}$ around its top-bottom axis). Participants had to decide as quickly as possible whether a pose was normal or not by pressing one of two buttons. Besides providing a more sensitive dependent measure, the use of the impossible poses allowed us to further explore the effects of long-term priming. Clearly, the very nature of the impossible poses not only makes them new to an observer, but also

$61 \%$ and $52 \%$ are different from 58\% in Experiment 1 and $50 \%$ in Experiment 2 (Table 1) for two reasons. First, only the mirror-image/right-facing and not the right-facing/mirror-image priming/prime pairs from Experiment 2 were included in the analysis. Second, "drinking from a bottle" in Experiment 1 was replaced by "polishing shoes" in Experiment 2. Thus, the data for these two stimuli were not taken into account. 
seriously counters general knowledge and beliefs about what human bodies look like. This means that the body-specific representational system will probably run into difficulties when trying to construct a proper global description of the stimulus and there will be no corresponding body model available for a simple match with whatever description the system finally comes up with. Consequently, if long-term priming effects result from the persistent activation or facilitated reactivation of global, high-level representations, no priming effects and therefore no difference between the same-view, different-view, and noprime condition should be observed for the impossible poses. This hypothesis is consistent with the results from an experiment conducted by Nilsson, Olofsson, and Nyberg (1992), who found no priming effects for distorted dynamic actions.

\section{Method}

Participants. Forty-eight undergraduate psychology students participated for course credit. All participants had normal or corrected-to-normal vision.

Stimuli and Apparatus. The stimuli were four sets of 42 gray-scale pictures. The first set consisted of normal, non-instrumental actions and body poses performed by one human model, facing approximately $80^{\circ}$ to the left of a straight-ahead view. The second set consisted of impossible poses. The above-waist part of the body was oriented $80^{\circ}$ and the below-waist part $260^{\circ}$ $\left(180^{\circ}+80^{\circ}\right)$ to the left of a straight-ahead view, or vice versa. They were constructed from a large variety of $80^{\circ}$ and $260^{\circ}$ poses, that were all different from the poses used in the first set. Finally, the pictures of the third and fourth set were mirror-images of the first and second set, respectively. Figure 3 shows examples from the four sets. All actions and poses for the first and second set were photographed in black-and-white, cut out, and, in the case of the impossible poses, rearranged. They were then glued on a white piece of paper and digitized with a Tamarack Telecom ArtiScan 6000C scanner. The mirror-images for the third and fourth set were created using the ATVista Tips software. In addition, there were 14 practice stimuli made from pictures taken from magazines. Half of these were normal, the other half were impossible poses. During the experiment, all stimuli were generated by a computer equipped with an ATVista graphics system and presented on a Barco CDCT 6351B screen. In a standing up pose the human body subtended approximately $16 \mathrm{~cm}$ on the screen $\left(10.7^{\circ}\right.$ with an $85 \mathrm{~cm}$ viewing distance).

Procedure. The two normal and the two impossible stimulus sets were each divided into six groups of seven stimuli, which were rotated across participants in such a way that each stimulus in priming and primed block appeared equally often in the same-view, the different-view, and the no-prime condition. 

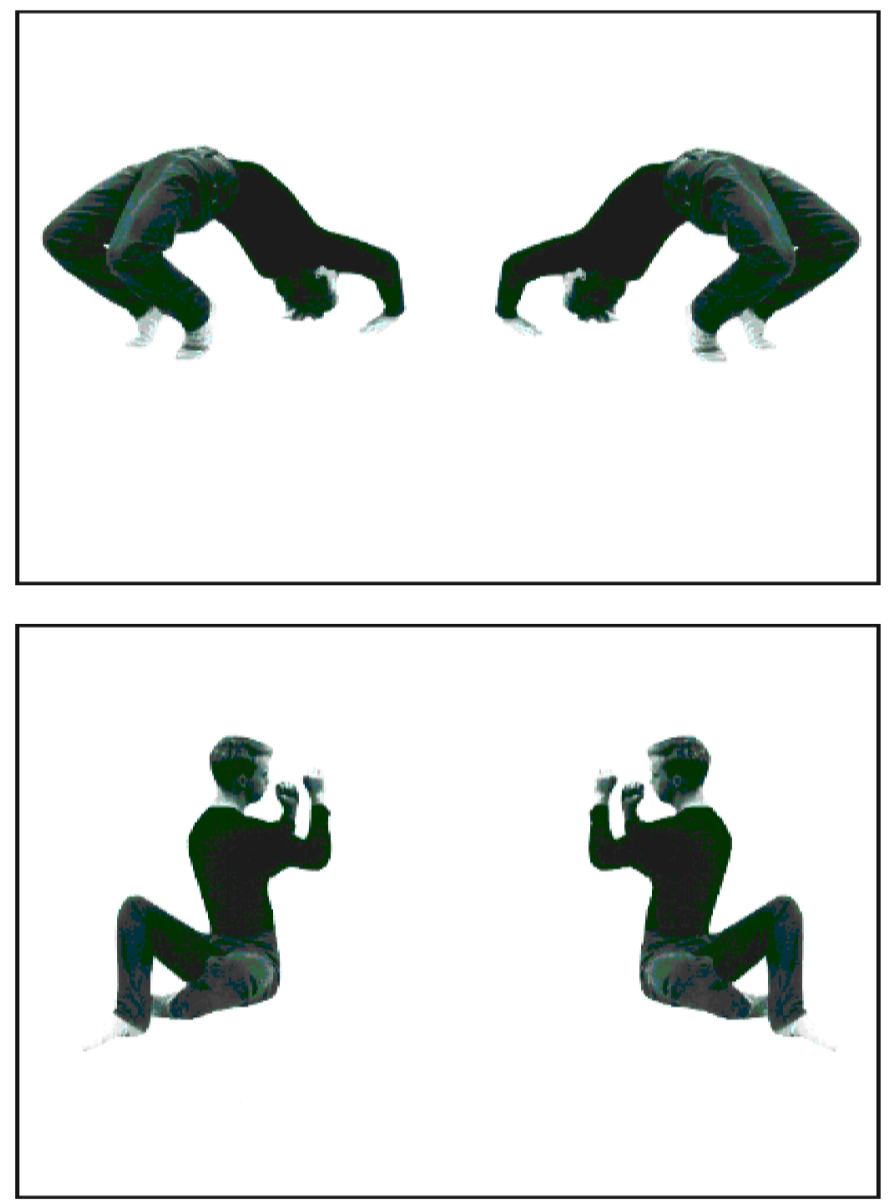

FIG. 3. A normal and an impossible side-view pose and their mirror-images.

Each participant saw 14 normal stimuli and 14 impossible stimuli in each condition. In the same-view condition half of the normal and half of the impossible stimuli were left-facing views and the other half were right-facing (mirror-image) views in the priming and the primed block. In the different-view condition, half of the stimuli were left-facing views in the priming block and their mirror-images in the primed block and the other half were mirror-images in the priming block and left-facing views in the primed block. In the no-prime condition, half of the stimuli in the primed block were left-facing views and the other half were right-facing (mirror-image) views. Hence, there were 56 priming stimuli (28 in the same-view and 28 in the different-view condition) and 84 
primed stimuli (28 same, 28 different, and 28 new stimuli). Participants started with the practice stimuli, immediately followed by a random presentation of the priming stimuli, a five-minute break and a random presentation of the primed stimuli. Each trial in the priming and primed block began with a warning sound and the appearance of a fixation cross in the middle of the screen. After $1 \mathrm{sec}$, the picture was presented and subjects had to decide whether the pictured action or body pose was normal or impossible by pressing one of two buttons as quickly as possible. Half of the participants pressed the right button to a normal pose and the left button to an impossible pose. This mapping was reversed for the other half of the participants. A correct response was followed by a high tone, an incorrect response by a low tone. The pictures disappeared from the screen after $2 \mathrm{sec}$ or as soon as the participant had pressed one of the buttons. The dependent measure was the time between stimulus onset and response.

\section{Results and Discussion}

The RTs that fell below a cutoff value of $200 \mathrm{msec}$ above a cutoff value of $1500 \mathrm{msec}$ (approximately 1\% of the data) and the RTs of the stimuli that were not identified correctly in either the priming or the primed block were discarded from the data set. All remaining RTs were entered into a subject and a stimulus repeated measures ANOVA with prime condition (same view, different view, or no prime) as within variable, stimulus type (normal or impossible body pose) as within (subject analysis) or between (stimulus analysis) variable, and subject group (or stimulus group) as between variable. Table 2 shows the mean RTs per condition.

The analyses revealed a statistically reliable main effect of stimulus type, $F_{1}(1,42)=29.21, \quad M S E=6761.34, \quad p<.0001 ; \quad F_{2}(1,72)=10.98, \quad M S E=$ $23806.81, p<.002$, indicating that normal body poses are generally recognized faster than impossible poses. Also, there was a main effect of prime condition, $F_{1}(2,84)=5.29, M S E=2443.17, p<.007$ in the subject analysis, but not in the

\section{TABLE 2}

Mean Reaction Times and Recognition Percentages of Primed Normal and Impossible Body Poses as a Function of Prime Condition (Experiment 3)

\begin{tabular}{|c|c|c|c|c|}
\hline \multirow[b]{3}{*}{ Prime Condition } & \multicolumn{4}{|c|}{ Stimulus Type } \\
\hline & \multicolumn{2}{|c|}{ Normal Poses } & \multicolumn{2}{|c|}{ Impossible Poses } \\
\hline & $(\mathrm{msec})$ & $(\%)$ & $(\mathrm{msec})$ & $(\%)$ \\
\hline Same view & 689 & 98.1 & 764 & 95.0 \\
\hline Mirror image & 713 & 96.6 & 764 & 95.7 \\
\hline No prime & 733 & 95.5 & 766 & 95.0 \\
\hline
\end{tabular}


stimulus analysis, $F_{2}(2,144)=1.02, M S E=3555.62, p>.30$, and a significant interaction effect between prime condition and stimulus type, $F_{1}(2,84)=6.06$, $M S E=1793.64, p<.004 ; F_{2}(2,144)=4.60, M S E=3555.62, p<.02$. Differences in prime condition means were tested using Dunn's multiple comparison procedure. The orientation-dependent priming effect was replicated: For the normal poses there was a significant difference between the same-view and the different-view prime condition, $t D_{1}=3.00, M S E=1568.40, p<.05$; $t D_{2}=3.10, M S E=1359.88, p<.05$, and between the same-view and the no-prime condition, $t D_{1}=4.78, M S E=2071.10, p<.01 ; t D_{2}=4.50, M S E=$ $1720.87, p<.01$. The relatively large $\mathrm{RT}$ difference between the different-view and the no-prime condition was not significant, $t D_{1}=2.46, M S E=1619.71$, n.s., $t D_{2}=2.11, M S E=1183.73$, n.s., suggesting that mirror-image primes do not produce reliable priming effects. For the impossible poses, no significant differences were found, $t D_{1}=0.01, M S E=2670.18 ; t D_{1}=0.20, M S E=$ $2409.57 ; t D_{1}=0.19, M S E=2371.47 ; t D_{2}=0.50, M S E=8353.87 ; t D_{2}=0.98$, $M S E=4579.93 ; t D_{2}=0.32, M S E=4135.42$ for the same-view/differentview, the same-view/no-prime, and the different-view/no-prime comparisons, respectively. Apparently, there was no priming in the case of the impossible poses, not even when an identical same-view picture was used as prime.

The percentage of correctly identified actions in the primed block was very high in all conditions. If there was a priming block error, the associated stimulus was not taken into account. Also, the data from five impossible stimuli that were not identified correctly by more than half of the subjects in the priming and in the primed block were discarded altogether. Inspection of the data in Table 2 shows that the pattern of the mean correct response percentages per condition is virtually identical to the pattern of RTs, thus ruling out the possibility of a speed-accuracy trade-off. The accuracy data were entered into a repeated measures subject analysis and a repeated measures stimulus analysis. Both analyses revealed no significant effects.

The findings of Experiment 3 again suggest that the representations that mediate the identification of normal actions and body postures are viewpoint specific: Same-view primes were more effective in decreasing identification times than mirror-image primes.

However, as mentioned in the Results and Discussion section of Experiment 2 , the observed priming effect could be pose specific rather than orientation specific. Again, there were more asymmetrical (27) than symmetrical (15) normal body poses in the stimulus set, but both categories were considered sufficiently large to conduct a GLM stimulus analysis with prime condition as within-stimuli variable and stimulus type (asymmetrical and symmetrical pose) and stimulus group as between-stimuli variables. There was a main effect of prime condition, $F_{2}(2,60)=8.69, M S E=1466.64, p<.0005$, but no interaction with stimulus type, $F_{2}(2,60)=0.78, M S E=1467.64, p>.40$, indicating that the same-view benefit generalized over both symmetrical and 
asymmetrical pose types. This result suggests that the observed priming effect is probably orientation specific and not pose or phase specific. Therefore, it can be concluded that action and posture recognition is indeed viewpoint dependent, even when different views consist of the same visible parts.

Although asymmetry of the part structure of human body postures does not explain the mirror-image disadvantage, the difference between the object perception and the action and posture perception experiments may still be accounted for by the fact that identification of actions and postures predominately involves the spatial relations or configuration of the parts rather than the parts themselves. Different postures are actually quite similar to each other. Apart from the fact that the stimulus objects in action or posture perception share the exact same body parts, there are many restrictions on the spatial relations between the parts as well. Lower limbs are always attached to upper limbs in the same way, upper limbs are always attached to the body in the same way, etc. All variation is within the limits of movement of the body parts. In this sense, action and posture identification is probably closer to subordinate-level than to basic-level identification. Several authors (e.g. Logothetis \& Sheinberg, 1996; Tarr \& Bülthoff, 1995) noticed that the former is more reliant on viewpoint-dependent mechanisms than the latter. When objects become increasingly similar across both parts and spatial relations, object recognition becomes more viewpoint specific (see also Edelman, 1995).

The results of Experiment 3 provide no direct evidence for an additional role of viewpoint-independent representations in the identification of human actions and body postures: The priming effect in the mirror-image prime condition in Experiment 2 (compared to the different-view condition from Experiment 1) was not unequivocally confirmed in Experiment 3. A similar discrepancy between the results from naming and decision tasks has been observed in the object perception literature. Naming generally seems to produce greater priming across different views than an object/non-object decision task (e.g. Srinivas, 1993, 1995). It has been suggested that this may be due to the fact that naming involves more semantic or associative components than decision tasks (Riddoch \& Humphreys, 1987). Identifying objects or actions and body postures as such may thus be achieved on the basis of high-level viewpoint-specific representations, whereas viewpoint-independent representations may be involved in stages that go beyond mere perception and identification.

The complete absence of priming in the perception of impossible poses has at least two important implications. First, this finding indirectly supports the idea that the orientation-specific priming effect in the case of the normal body poses is indeed based on the reactivation of global high-level representations. Actually, the viewpoint-dependent nature of any lower-level procedure or representation that makes up an earlier phase of the identification process could in principle be responsible for an orientation-specific priming effect (Verfaillie, 
1992). In that case, however, the impossible poses should have profited equally from an identical same-view prime as the normal poses. After all, both stimulus types were made up of the same parts and there is no immediate reason to assume that low-level processing would in any way be different or more difficult for the impossible poses.

Second, Schacter and colleagues reported long-term priming effects for completely novel, rigid three-dimensional objects, but not for comparable impossible objects (e.g. Schacter et al., 1990, Schacter, Cooper, Delaney, Peterson, \& Tharan, 1991). Contrary to their first finding (possible-object priming) and in line with their second finding (no impossible-object priming), we found no evidence for priming in the case of the impossible poses, which are not only new to the observer, but have a possible 3D interpretation as well. In this sense, they are equivalent to Schacter and colleagues' possible objects rather than their impossible objects. This might suggest that human body posture recognition is indeed accomplished by a specialized processing system (Reed \& Farah, 1995). On the one hand, it will probably be necessary to activate the body-specific representational system to be able to decide that a posture is impossible. This might take some more time and effort than in the case of the normal poses, which explains the overall slower recognition times. On the other hand, it may be too difficult for the body-specific system to form proper global descriptions for poses that seriously counter general knowledge about what human bodies look like. If the system doesn't succeed, there can be no reactivation in the primed block and no priming effects.

However, there is another, less interesting explanation for the absence of priming effects in the case of impossible body poses, which is somewhat analogous to Ratcliff and McKoon's (1995) objections to Schacter and colleagues' interpretation of their priming data (e.g. 1990, 1991). In possible/impossible decision tasks, participants may have a tendency to respond "possible" to studied or familiar stimuli. Such a response bias would facilitate responses to possible poses and hamper responses to impossible poses. Combined with an explicit memory (see Ratcliff \& McKoon, 1995) or implicit memory (e.g. view-specific priming) component, this could lead to robust positive effects for the possible and to null effects for the impossible poses. However, inspection of the error data makes it rather unlikely that this bias played a significant role in Experiment 3. The extremely low error rates and the fact that the error analysis revealed no significant differences between primed and unprimed poses and between possible and impossible poses indicate that participants experienced no uncertainty in making possible/impossible decisions, even for stimuli that were never encountered before. They were able to precisely place their criterion and separate possible from impossible poses, which means that response biases probably remained the same regardless of whether the poses were primed or not. A similar argument was advanced by Williams and Tarr (1997, Exp. 3). By presenting stimuli in an object-decision task until participants 
responded (which was also the case in our experiment), they were able to equate accuracy rates and they found reliable RT priming effects for possible poses only. From this finding, Williams and Tarr concluded that differences between studied and unstudied stimuli are not caused by a bias to respond "possible". Of course, it is not completely inconceivable that response biases influence RTs, even in the absence of any accuracy effects. Therefore, in order to rule out this explanation altogether, further experimentation will be needed. Participants could for instance be instructed to respond "possible" to the impossible poses from Experiment 3 and "impossible" to a different kind of impossible poses (e.g. poses where limbs stick through the body or through each other). If no priming effects were to be observed in the "possible" response condition, the bias explanation could be discarded. Such a result would also provide a strong argument for the hypothesis that human actions and body poses are processed by a separate body-specific representational system.

\section{EXPERIMENT 4}

The previous experiments support the idea that the representations mediating the identification of human actions and body postures are viewpoint specific. The main purpose of Experiment 4 was to examine more closely how specific or how sharply tuned to a particular view these representations are. Perrett et al.'s (e.g. 1989, 1991) single-cell recordings in the temporal cortex of monkeys showed that the activation level of face cells and body-specific cells with view-specific coding dropped to half maximal with orientation differences between $45^{\circ}$ and $70^{\circ}$. This finding suggests that view tuning is rather sharp. In the experiment described here, view tuning was examined with orientation differences ranging from $0^{\circ}$ to $60^{\circ}$. The experiment consisted of six prime conditions: The priming and primed actions or poses differed by $0^{\circ} 15^{\circ} 30^{\circ} 45^{\circ}$ or $60^{\circ}$. As spelled out in detail in the Method section, great care was taken that as many body parts as possible were visible in all views.

As in Experiment 3, the experimental task was a forced-choice reaction time task. Half of the stimuli were normal poses and the other half were impossible poses. The different view manipulations were made for both stimulus types. On the basis of the results from Experiment 3, it was expected that there would be no priming in the case of the impossible poses.

\section{Method}

Participants. Eighty-four undergraduate psychology students participated for course credit. All participants had normal or corrected-to-normal vision. 
Stimuli and Apparatus. The stimuli were 20 sets of 24 gray-scale pictures. The first five sets consisted of 24 normal actions and body poses oriented $15^{\circ}$, $30^{\circ}, 45^{\circ}, 60^{\circ}$, and $75^{\circ}$ to the right of a straight-ahead view. The second five sets consisted of 24 normal actions and body poses oriented $195^{\circ}, 210^{\circ}, 225^{\circ}, 240^{\circ}$ and $255^{\circ}$ to the right of a straight-ahead view. Hence, there were 48 normal actions, 24 of which were depicted from five different front views and 24 of which were depicted from five different rear views. The remaining 10 sets consisted of impossible poses. The below-waist part of the body was oriented $15^{\circ}, 30^{\circ}$, $45^{\circ}, 60^{\circ}$, and $75^{\circ}$ to the right of a straight-ahead view in the first five sets and $195^{\circ}, 210^{\circ}, 225^{\circ}, 240^{\circ}$, and $255^{\circ}$ in the other five sets. The above-waist part of the body was always oriented $180^{\circ}$ further to the right (this means from $195^{\circ}$ to $255^{\circ}$ and from $15^{\circ}$ to $75^{\circ}$, respectively). The 48 impossible poses were all generated from different poses than the 48 normal poses. Care was taken that as many body parts as possible were visible in all views. The construction of the poses and the choice for a particular stimulus category (normal $15^{\circ}-75^{\circ}$, normal $195^{\circ}-255^{\circ}$, impossible below-waist $15^{\circ}-75^{\circ}$ and impossible below-waist $195^{\circ}-255^{\circ}$ ) for every individual pose was constrained by this criterion. The same body parts were visible in all views in approximately one-third of the stimuli. If parts of a limb were occluded in some views but not in others, it was mostly only the upper or the lower part. In these cases the position of the occluded parts could often be inferred from the position of the visible upper or lower parts of the same limb (e.g. the left upper leg in the $15^{\circ}$ view of the first pose in Figure 4). The priming experiments of Williams and Tarr (1997) provide some tentative evidence that the visual system might have the capacity to generate missing parts: Participants seemed to be able to fill in 3D structure behind an occluder. Finally, if the position of a part of a limb was ambiguous or if a limb was completely occluded, it was always symmetrical to the contralateral visible limb (e.g. the right arm in the $45^{\circ}$ view of the second pose in Figure 4). It has been shown that symmetry is a powerful constraint in the completion of occluded object parts (Van Lier \& Wagemans, in press).

All normal and impossible poses were first created using the Poser software (Fractal Design, 1996) and then improved with a graphics software package. There were 14 practice stimuli. These were the same as in Experiment 3. During the experiment, stimuli were generated by a computer equipped with a Trident VGA graphics card and presented on a 15-inch computer screen. In a standing up pose the human body subtended approximately $12 \mathrm{~cm}$ on the screen $\left(10.5^{\circ}\right.$ with a $65 \mathrm{~cm}$ viewing distance).

Procedure. The experiment again consisted of a priming and a primed block. All stimuli in the primed block were presented in the $75^{\circ}$ or the $255^{\circ}$ view. The stimuli in the priming block were either in the same view $\left(75^{\circ}\right.$ or $\left.255^{\circ}\right)$, a $15^{\circ}$-different view $\left(60^{\circ}\right.$ or $\left.240^{\circ}\right)$, a $30^{\circ}$-different view $\left(45^{\circ}\right.$ or $215^{\circ}$ ), a $45^{\circ}$-different view $\left(30^{\circ}\right.$ or $\left.210^{\circ}\right)$, a $60^{\circ}$-different view $\left(15^{\circ}\right.$ or $\left.195^{\circ}\right)$, or there 

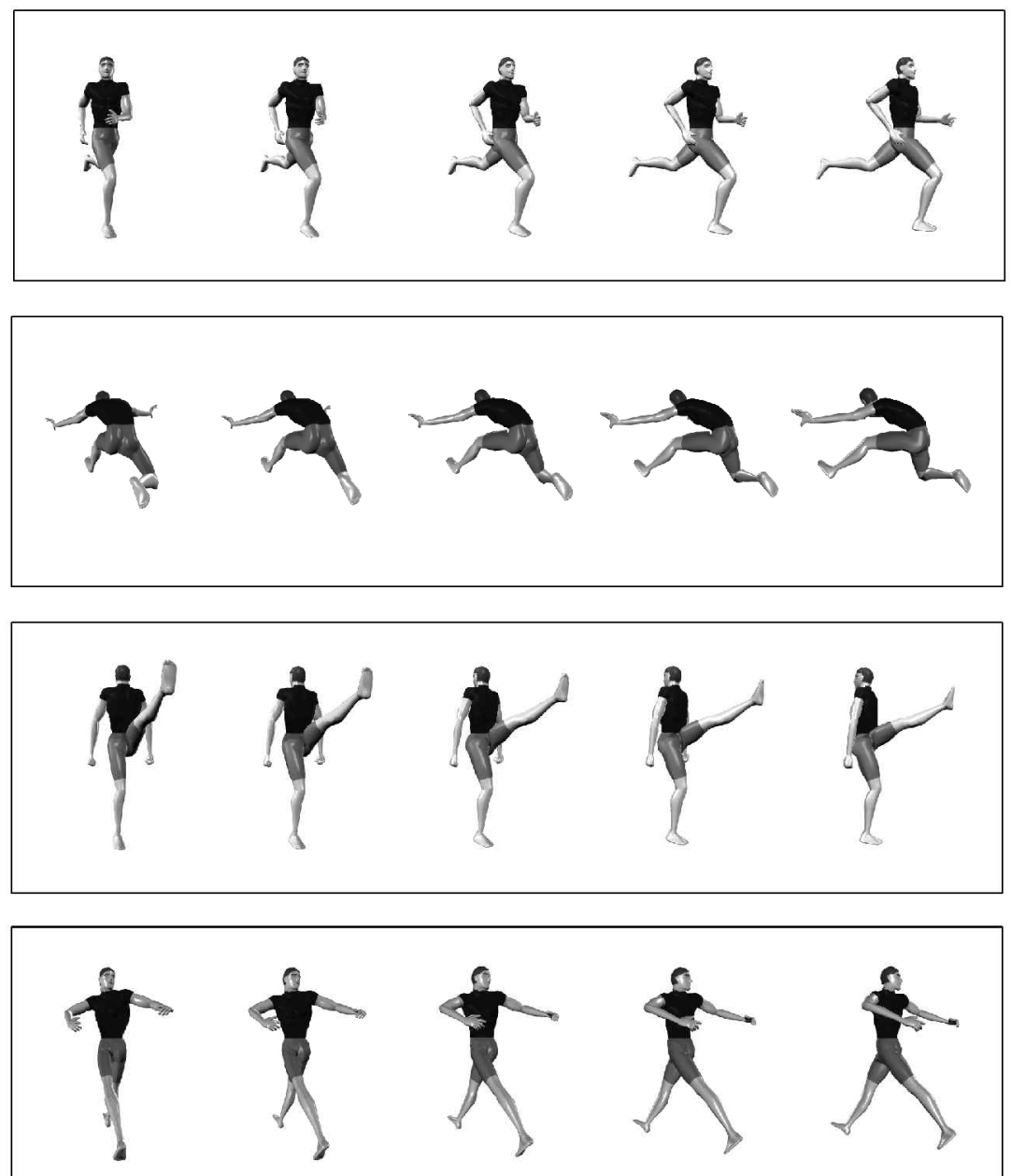

FIG. 4. Two normal and two impossible body poses oriented from $15^{\circ}$ to $75^{\circ}$ or from $195^{\circ}$ to $255^{\circ}$ to the right of a straight-ahead view.

was no prime. The ten normal and the ten impossible stimulus sets were each divided into six groups of four stimuli, which were rotated across participants in such a way that each stimulus appeared equally often in each prime condition. There were 16 stimuli in each prime condition. Eight out of 16 were normal and eight out of 16 were impossible. Four out of eight normal and impossible poses were oriented from $15^{\circ}$ to $75^{\circ}$ to the right and four were 
oriented from $195^{\circ}$ to $255^{\circ}$. All participants started the experiment with the practice stimuli, immediately followed by a random presentation of 80 priming stimuli, a short break, and a random presentation of 96 primed stimuli. The trials in the priming and primed block all began with a warning sound. After $500 \mathrm{msec}$ the picture was presented and participants had to decide whether it was a normal or impossible action or body pose by pressing one of two buttons as quickly as possible. The rest of the procedure was the same as in Experiment 3.

\section{Results and Discussion}

The RTs that fell below a cutoff value of $200 \mathrm{msec}$ or above a cutoff value of $1400 \mathrm{msec}$ (approximately $1 \%$ of the data) and the RTs of the stimuli that were not identified correctly in either the priming or the primed block were discarded from the RT data set. ${ }^{2}$ All remaining RTs were entered into a subject and a

${ }^{2}$ By administering an identification task in the priming block and by removing the primed stimulus when there was an error in the corresponding priming stimulus, it was ensured that prior identification had indeed occurred for all the remaining stimuli in the primed dataset. However, a consequence of the priming block task was that participants did not necessarily see all priming stimuli for an equally long time. Hence, if stimuli in certain views were consistently identified more slowly in the priming block than stimuli in other views, this might influence priming levels independent of any view-specific effects. Therefore, a repeated measures subject and stimulus analysis was conducted on the RT priming data. In the subject analysis, the interaction between stimulus type (normal or impossible poses) and prime condition $\left(60^{\circ}\right.$-different, $45^{\circ}$-different, $30^{\circ}$-different, $15^{\circ}$-different, and same views as in the primed block) was marginally significant, $F_{1}(4,312)=2.39, M S E=5546.68, p<.06$. For the normal poses there was a significant effect of prime condition in the subject analysis, $F_{1}(4,312)=2.68, M S E=4366.26, p<.04$, but Dunn's multiple comparison procedure revealed no significant differences between mean RTs $(815 \mathrm{msec}$, $828 \mathrm{msec}, 805 \mathrm{msec}, 810 \mathrm{msec}$ and $797 \mathrm{msec}$ in the $60^{\circ}$-, $45^{\circ}$-, $30^{\circ}$-, $15^{\circ}$-different, and same-view condition, respectively). It seems unlikely that these small non-significant differences were responsible for the primed block results. First, the mean RT was lowest in the same-view condition $\left(75^{\circ}\right.$ and $255^{\circ}$ views). This means that the largest priming effect was obtained in the condition where participants saw the stimulus for the smallest amount of time. Also, identification times in this condition were only $8 \mathrm{msec}$ faster than identification times in the $30^{\circ}$-different view condition $\left(45^{\circ}\right.$ and $225^{\circ}$ views), and the latter condition produced no priming at all. For the impossible poses, both analyses revealed an effect of prime condition, $F_{1}(4,312)=4.73, M S E=6622.18$, $p<.002 ; F_{2}(4,164)=2.68, M S E=5041.95, p<.04$. RT means were $913 \mathrm{msec}, 878 \mathrm{msec}$, $867 \mathrm{msec}, 877 \mathrm{msec}$ and $871 \mathrm{msec}$ in the $60^{\circ}$-, $45^{\circ}$-, $30^{\circ}$-, $15^{\circ}$-different, and same-view condition. The $60^{\circ}$-different view condition differed significantly from the $30^{\circ}$-different view condition. Hence, impossible poses were recognized faster when seen in halfway views $\left(45^{\circ}\right.$ and $225^{\circ}$ in the $30^{\circ}$-different view condition) than in front or back views $\left(15^{\circ}\right.$ and $195^{\circ}$ in the $60^{\circ}$-different view condition), but this difference in the priming block did influence priming levels in the primed block. 
stimulus repeated measures ANOVA with prime condition (same view, $15^{\circ}$-different view, $30^{\circ}$-different view, $45^{\circ}$-different view, $60^{\circ}$-different view, or no prime) as within variable, stimulus type (normal or impossible body pose) as within (subject analysis) or between (stimulus analysis) variable, and subject (or stimulus) group as between variable. Table 3 shows the mean RTs per condition.

The ANOVA revealed a main effect of stimulus type, $F_{1}(1,78)=87.39$, $M S E=14882.68, p<.0001 ; F_{2}(1,83)=30.21, M S E=26012.11, p<.0001$, which shows that normal poses are identified faster than impossible poses. There was also a main effect of prime condition, $F_{1}(5,390)=2.59$, $M S E=3788.30, p<.03 ; F_{2}(5,415)=2.85, M S E=2587.63, p<.02$, and no statistically reliable interaction between stimulus type and prime condition, $F_{1}(5,390)=1.02, M S E=3452.74, p>.40 ; F_{2}(5,415)=1.34, M S E=2587.63$, $p>.20$.

Although the interaction between stimulus type and prime condition was not significant, we decided on the basis of the results of Experiment 3 to test for differences between the prime conditions and the no-prime baseline condition and for trends for the normal and impossible actions and poses separately.

A strong orientation-dependent priming effect was observed for the normal poses. First, a reliable priming effect was obtained only in the same-view prime condition. Dunnett's multiple comparison test between the no-prime baseline condition and each of the prime conditions (Kirk, 1995, pp.134-137) revealed a significant difference between the no-prime and the same-view prime condition, $t D N_{1}=3.34, M S E=2770.49, p<.01$, but not between the no-prime and the different-view prime conditions, $t D N_{1}=2.00, M S E=2412.54$, n.s. for the $15^{\circ}$-different view condition, $t D N_{1}=0.69, M S E=3136.58$, n.s. for the

\section{TABLE 3}

Mean Reaction Times and Recognition Percentages of Primed Normal and Impossible Body Poses as a Function of Prime Condition (Experiment 4)

\begin{tabular}{|c|c|c|c|c|}
\hline \multirow[b]{3}{*}{ Prime Condition } & \multicolumn{4}{|c|}{ Stimulus Type } \\
\hline & \multicolumn{2}{|c|}{ Normal Poses } & \multicolumn{2}{|c|}{ Impossible Poses } \\
\hline & (msec) & $(\%)$ & $(\mathrm{msec})$ & $(\%)$ \\
\hline Same view & 660 & 97.5 & 745 & 95.5 \\
\hline $15^{\circ}$-different view & 672 & 97.1 & 748 & 95.3 \\
\hline $30^{\circ}$-different view & 681 & 96.6 & 756 & 93.8 \\
\hline $45^{\circ}$-different view & 682 & 95.4 & 742 & 93.5 \\
\hline $60^{\circ}$-different view & 687 & 95.5 & 749 & 95.4 \\
\hline No prime & 687 & 93.8 & 761 & 94.3 \\
\hline
\end{tabular}


$30^{\circ}$-different view condition, $t D N_{1}=0.60, M S E=3216.68$, n.s. for the $45^{\circ}$ different view condition, and $t D N_{1}=0.03, M S E=5360.53$, n.s. for the $60^{\circ}$ different view condition. This result was confirmed in the stimulus analysis. There was a reliable difference between the no-prime and the same-view prime condition, $t D N_{2}=3.55, M S E=1442.47, p<.01$, but not between the no-prime and the different-view prime conditions.

Second, there was a significant linear trend in the subject analysis, $F_{1}(1,78)=15.14, M S E=2588.97, p<.0007$, indicating that priming gradually increased when the view difference between the priming and the primed pose decreases. However, in addition to the linear trend, $F_{2}(1,42)=19.45$, $M S E=1245.90, p<.0001$, the stimulus analysis also revealed a quadratic trend, $F_{2}(1,42)=5.21, M S E=1670.22, p<.03$. The presence of this trend component and the absence of any other significant higher order components suggests that the priming effect quickly stabilizes. In fact, Table 3 shows that stabilization already occurs in the $30^{\circ}$-different view condition.

No significant differences were observed in the case of the impossible poses, $t D N_{1}=1.85, M S E=3297.08 ; t D N_{1}=1.21, M S E=4877.26 ; t D N_{1}=0.52$, $M S E=4343.45 ; t D N_{1}=2.06, M S E=3490.84 ; t D N_{1}=1.27, M S E=3892,12$ for the same-view/no-prime, the $15^{\circ}$-different view/no-prime, $30^{\circ}$-different view/no-prime, the $45^{\circ}$-different view/no-prime, and the $60^{\circ}$-different view/no-prime comparison in the subject analysis. Also, there were no reliable trends.

As in Experiment 3, the percentage of correctly identified actions or poses in the primed block exceeded $90 \%$ in all conditions. The stimuli that were not categorized correctly in the priming block were not taken into account. Also, there were three impossible stimuli, which were identified by less than half of the subjects in the priming and in the primed block. These stimuli were discarded from the dataset altogether. The pattern of the accuracy data again mirrors the pattern of the RT data (see Table 3). There was a significant main effect of stimulus type and a marginally significant main effect for prime condition in the subject analysis, $F_{1}(1,78)=4.69, M S E=0.0097, p<.04$, and $F_{1}(5,390) 0=$ $2.19, M S E=2.7937, p<.06$, no significant main effect for stimulus type and a significant main effect for prime condition in the stimulus analysis, $F_{2}(1,81)=1.34, M S E=0.0145, p>.20$, and $F_{2}(5,405)=2.67, M S E=0.0035$, $p<.03$, and no significant interaction between stimulus type and prime condition in either analysis, $F_{1}(5,390)=1.01, \quad M S E=0.0068, p>.40$; $F_{2}(5,405)=0.98, M S E=0.0035, p>.40$. Despite the absence of a significant interaction effect, it was decided on the basis of the RT results to examine the data for the normal and impossible poses separately. For the normal poses, Dunnett's multiple comparison test between the no-prime baseline condition and each of the prime conditions revealed a significant difference between the no-prime and the same-view prime condition in both analyses, $t D N_{1}=3.39$, 
$M S E=0.0052, p<.01 ; t D N_{2}=3.15, M S E=0.0032, p<.05$, and between the no-prime and the $15^{\circ}$-different-view prime condition in the stimulus analysis only, $t D N_{2}=3.16, M S E=0.00036, p<.05$. All other differences were not significant. Also, a linear trend was observed, $F_{1}(1,78)=10.99, M S E=0.0066$, $p<.002 ; F_{2}(1,42)=15.35, M S E=0.0031, p<.0003$. There were no significant effects in the impossible-pose condition.

For the normal human actions and body postures, reliable priming was obtained mainly in the same-view prime condition. Reaction times and errors in the primed block increased rapidly with increasing orientation differences in the priming block: The priming effect dropped halfway in the $15^{\circ}$-different view prime condition and was nearly non-existent when view differences exceeded $30^{\circ}$. These results suggest that the viewpoint-specific representations involved in the identification of normal human actions and body postures are very sharply tuned.

As expected, there were no reliable priming effects in the case of the impossible poses. Experiment 4 therefore supports the idea that the orientation-specific nature of the priming effect in the case of the normal poses indeed originates from the orientation-specific nature of global high-level representations.

\section{GENERAL DISCUSSION}

The perception of a normal human action or body posture facilitates identification of the same action or posture on a later occasion, provided that priming and primed pose share the same in-depth orientation and left-right reflection. There is no strong evidence that recognizing a posture after viewing exactly the same posture in a different orientation is faster than recognizing it without a preceding prime. Although there is a gradual increase in identification performance when increasingly similar orientations are seen before, this seems to be the case only for orientation differences under $30^{\circ}$. These findings suggest that the representations that mediate action and body identification are sharply tuned to a particular perspective view.

People can very rapidly identify a human body as a basic-level object. To this end, the visual system has to abstract away from the particular configuration of the body parts. Irrespective of the 3D arrangement of parts, the stimulus object is classified as a human body. Our experiments tap into other processes. Participants have to classify the 3D configuration rather than transcending it. The arrangement of body parts determines which action label the stimulus receives (Experiments 1 and 2) and whether the stimulus is a normal pose or not (Experiments 3 and 4). In this sense, action or pose classification is more akin to subordinate-level object identification than to basic-level object identification. 
From this perspective, the extreme orientation specificity that was observed in the present experiments is not at all surprising: As already mentioned, it has been shown before that subordinate-level object recognition, or more generally, recognition of similar objects (e.g. objects that differ in the spatial relations between parts only) is highly viewpoint-dependent (e.g. Edelman, 1995; Tarr \& Bülthoff, 1995).

\section{REFERENCES}

Bertenthal, B.I., \& Pinto, J. (1993). Complementary processes in the perception and production of human movements. In E. Thelen \& L. Smith (Eds.), Dynamical approaches to development: Vol. 2. Applications (pp. 209-239). Cambridge, MA: Bradford Books.

Biederman, I. (1987). Recognition-by-components: A theory of human image understanding. Psychological Review, 94, 115-147.

Biederman, I., \& Cooper, E.E. (1991). Evidence for complete translational and reflectional invariance in visual object priming. Perception, 20, 585-593.

Biederman, I., \& Gerhardstein, P.C. (1993). Recognizing depth-rotated objects: Evidence and conditions for three-dimensional viewpoint invariance. Journal of Experimental Psychology: Human Perception and Performance, 19, 1162-1182.

Biederman, I., \& Gerhardstein, P.C. (1995). Viewpoint-dependent mechanisms in visual object recognition: Reply to Tarr and Bülthoff (1995). Journal of Experimental Psychology: Human Perception and Performance, 21, 1506-1514.

Cooper, L.A., Schacter, D.L., Ballesteros, S., \& Moore, C. (1992). Priming and recognition of transformed three-dimensional objects: Effects of size and reflection. Journal of Experimental Psychology: Learning, Memory, and Cognition, 18, 43-57.

Cooper, L.A., \& Shepard, R.N. (1975). Mental transformations in the identification of left and right hands. Journal of Experimental Psychology: Human Perception and Performance, 104, 48-56.

Edelman, S. (1995). Class similarity and viewpoint invariance in the recognition of 3D objects. Biological Cybernetics, 72, 207-220.

Ellis, R., \& Allport, D.A. (1986). Multiple levels of representation for visual objects: A behavioural study. In A.G. Cohn \& J.R. Thomas (Eds.), Artificial intelligence and its applications (pp 245-257). Chichester, Wiley.

Ellis, R., Allport, D.A., Humphreys, G.W., \& Collis, J. (1989). Varieties of object constancy. Quarterly Journal of Experimental Psychology, 41A, 775-796.

Farah, M.L, Meyer, M.M., \& McMullen, P.A. (1996). The living/nonliving dissociation is not an artifact: Giving an a priori implausible hypothesis a strong test. Cognitive Neuropsychology, 13, 137-154.

Farah, M.L, McMullen, P.A., \& Meyer, M.M. (1991). Can recognition of living things be selectively impaired? Neuropsychologia, 29, 185-193.

Fractal Design Corporation (1996). Poser. [Computer software]. Aptos, CA.

Hayward, W.G., \& Tarr, M.J. (1997). Testing conditions for viewpoint invariance in object recognition. Journal of Experimental Psychology: Human Perception and Performance, 23, 1511-1521,

Jolicoeur, P. (1985). The time to name disoriented natural objects. Memory and Cognition, 13, 289-303. 
Jolicoeur, P. (1988). Mental rotation and the identification of disoriented objects. Canadian Journal of Psychology, 42, 461-478.

Jolicoeur, P. (1990). Identification of disoriented objects: A dual-systems theory. Mind and Language, 5, 387-410.

Kirk, R.E. (1995). Experimental design: Procedures for the behavioral sciences. Pacific Grove, CA: Brooks/Cole.

Kurbat, M.H. (1997). Can the recognition of living things really be selectively impaired? Neuropsychologia, 6, 813-827.

Lawson, R., \& Humphreys, G.W. (1996). View specificity in object processing: Evidence from picture matching. Journal of Experimental Psychology: Human Perception and Performance, 22, 395-416.

Lawson, R., Humphreys, G.W., \& Watson, D.G. (1994). Object recognition under sequential viewing conditions: Evidence for viewpoint-specific recognition procedures. Perception, 23, 595-614.

Logothetis, N.K., Sheinberg, D.L. (1996). Visual object recognition. Annual Review of Neuroscience, 19, 577-621.

Marr, D. (1982). Vision: A computational investigation into the human representation and processing of visual information. San Francisco: Freeman.

Marr, D., \& Nishihara, H.K. (1978). Representation and recognition of the spatial organization of three-dimensional shapes. Proceedings of the Royal Society of London, B200, 269-294.

Marshall, E., \& Walker, P. (1987). Visual memory for pictorial stimuli in a serial choice reaction-time task. British Journal of Psychology, 78, 213-231.

Nilsson, L.-G., Olofsson, U., \& Nyberg, L. (1992). Implicit memory of dynamic information. Bulletin of the Psychonomic Society, 30, 265-267.

Ogden, J.A. (1985). Autotopagnosia: Occurrence in a patient without nominal aphasia and with an intact ability to point to parts of animals and objects. Brain, 108, 1009-1022.

Olofsson, U., Nyberg, L., \& Nilsson, L.-G. (1997). Priming and the recognition of human motion patterns. Visual Cognition, 4, 373-382.

Parsons, L.M. (1987a). Imagined spatial transformations of one's body. Journal of Experimental Psychology: General, 116, 172-191.

Parsons, L.M. (1987b). Imagined spatial transformations of one's hands and feet. Cognitive Psychology, 19, 178-241.

Parsons, L.M. (1994). Temporal and kinematic properties of motor behavior reflected in mentally simulated action. Journal of Experimental Psychology: Human Perception and Performance, 20, 709-730.

Parsons, L.M., Fox, P.T., Downs, J.H., Glass, T., Hirsch, T.B., Martin, C.C., Jerabek, P.A., \& Lancaster, J.L. (1995). Use of implicit motor imagery for visual shape discrimination as revealed by PET. Nature, 375, 54-58.

Perrett, D.L., Harries, M.H., Bevan, R., Thomas, S., Benson, P.J., Mistlin, A.J., Chitty, A.J., Hietanen, J.K., \& Ortega, J.E. (1989). Frameworks of analysis for the neural representation of animate objects and actions. Journal of Experimental Biology, 146, 87-113.

Perrett, D.I., Oram, M.W., Harries, M.H., Bevan, R., Hietanen, J.K., Benson, P.L, \& Thomas, S. (1991). Viewer-centred and object-centred encoding of heads analysed at the single cell level in the temporal cortex of the rhesus macaque. Experimental Brain Research, 86, 159-173.

Ratcliff, R., \& McKoon, G. (1995). Bias in the priming of object decisions. Journal of Experimental Psychology: Learning, Memory, and Cognition, 21, 754-767.

Reed, C.L., \& Farah, M.J. (1995). The psychological reality of the body schema: A test with normal participants. Journal of Experimental Psychology: Human Perception and Performance, 21, 334-343. 
Riddoch, M.J., \& Humphreys, G.W. (1987). Picture naming. In G.W. Humphreys \& M.J. Riddoch (Eds.), Visual object processing: A cognitive neuropsychological approach (pp. 107-143). Hove, UK: Lawrence Erlbaum Associates Ltd.

Schacter, D.L., Cooper, C.A., \& Delaney, S.M. (1990). Implicit memory for unfamiliar objects depends on access to structural descriptions. Journal of Experimental Psychology: General, 119, 5-24.

Schacter, D.L., Cooper, L.A., Delaney, S.M., Peterson, M.A., \& Tharan, M. (1991). Implicit memory for possible and impossible objects: Constraints on the construction of structural descriptions. Journal of Experimental Psychology: Learning, Memory, and Cognition, 17, 3-19.

Shiffrar, M., \& Freyd, J.J. (1990). Apparent motion of the human body. Psychological Science, $1,257-264$.

Shiffrar, M., \& Freyd, J.J. (1993). Timing and apparent motion path choice with human body photographs. Psychological Science, 4, 379-384.

Shiffrar, M., Lichtey, L., \& Heptulla Chatterjee, S.H. (1997). The perception of biological motion across apertures. Perception and Psychophysics, 59, 51-59.

Sirigu, A., Grafman, L., Bressler, K., \& Sunderland, T. (1991). Multiple representations contribute to body-knowledge processing: Evidence from a case of autotopagnosia. Brain, 114, $629-642$.

Srinivas, K. (1993). Perceptual specificity in nonverbal priming. Journal of Experimental Psychology: Learning, Memory, and Cognition, 19, 582-602.

Srinivas, K. (1995). Representation of rotated objects in explicit and implicit memory. Journal of Experimental Psychology: Learning, Memory, and Cognition, 21, 1019-1036.

Sumi, S. (1984). Upside-down presentation of the Johansson moving light-spot pattern. Perception, 13, 283-286.

Tarr, M.J. (1995). Rotating objects to recognize them: A case study of the role of mental transformations in the recognition of three-dimensional objects. Psychonomic Bulletin and Review, 2, 55-82.

Tarr, M.J., \& Bülthoff, H.H. (1995). Is human object recognition better described by geon structural descriptions or by multiple views? Comment on Biederman and Gerhardstein (1993). Journal of Experimental Psychology: Human Perception and Performance, 21, 1494-1505.

Tarr, M.J., \& Pinker, S. (1989). Mental rotation and orientation-dependence in shape recognition. Cognitive Psychology, 21, 233-282.

Tarr, M.J., \& Pinker, S. (1990). When does human object recognition use a viewer-centered reference frame? Psychological Science, 1, 253-256.

Van Lier, R., \& Wagemans, J. (in press). From images to objects: Global and local completions of self-occluded parts. Journal of Experimental Psychology: Human Perception and Performance.

Verfaillie, K. (1992). Variant points of view on viewpoint invariance. Canadian Journal of Psychology, 46, 215-235.

Verfaillie, K. (1993). Orientation-dependent priming effects in the perception of biological motion. Journal of Experimental Psychology: Human Perception and Performance, 19, 992-1013.

Verfaillie, K. (1997). Transsaccadic memory for the egocentric and allocentric position of a biological-motion walker. Journal of Experimental Psychology: Learning, Memory, and Cognition, 23, 739-760.

Verfaillie, K., De Troy, A., \& Van Rensbergen, J. (1994). Transsaccadic integration of biological motion. Journal of Experimental Psychology: Learning, Memory and Cognition, 20, 649-670. 
Wachsmuth, M.W., Oram, M.W., \& Perrett, D.I. (1994). Recognition of objects and their component parts: Responses of single units in the temporal cortex of the macaque. Cerebral Cortex, 5, 509-522.

Williams, P., \& Tarr, M.J. (1997). Structural processing and implicit memory for possible and impossible figures. Journal of Experimental Psychology: Learning, Memory, and Cognition, 23, 1344-1361

Manuscript received September 1997 Revised manuscript received November 1998 
Copyright of Visual Cognition is the property of Psychology Press (T\&F) and its content may not be copied or emailed to multiple sites or posted to a listserv without the copyright holder's express written permission. However, users may print, download, or email articles for individual use. 